\title{
Aptidão cardiorrespiratória de adolescentes de Florianópolis, SC
}

\author{
Daniel Giordani Vasques ${ }^{1}$, Kelly Samara da Silva ${ }^{1}$ e Adair da Silva Lopes ${ }^{1}$
}

\section{RESUMO}

Níveis adequados de aptidão cardiorrespiratória durante a adolescência estão diretamente relacionados a um estilo de vida saudável na fase adulta. Este estudo objetivou identificar o comportamento da aptidão cardiorrespiratória durante a adolescência e descrever a prevalência de adolescentes que atenderam e não atenderam o critério recomendado para a saúde por sexo, idade e nível socioeconômico (NSE). A amostra foi composta de 963 adolescentes (513 moças e 450 rapazes), com idades de 10 a 15 anos. $\mathrm{Na}$ coleta de dados, foi aplicado um questionário para obtenção do NSE $(A+B, C, D+E)$. Para análise da aptidão cardiorrespiratória, foi realizado o teste PACER (Progressive Aerobic Cardiovascular Endurance Run). Consideraram-se como parâmetros de desempenho cardiorrespiratório o número de voltas realizadas e o consumo máximo de oxigênio $-\dot{V} \mathrm{O}_{2 \max }(\mathrm{ml} / \mathrm{kg} / \mathrm{min})$. Na análise estatística, foram utilizadas médias e desvios padrões, análise de variância two-way com teste post-hoc de Tukey, e teste qui-quadrado $(p<0,05)$. Enquanto o número de voltas aumentou dos 10 aos 15 anos, o $\dot{\mathrm{V}} \mathrm{O}_{2 \max }$ diminuiu, não havendo diferenças entre os sexos. Os escolares dos NSE menos favorecidos $(D+E)$ obtiveram menor aptidão do que os demais. Identificou-se que $68 \%$ dos rapazes e $37,8 \%$ das moças não atingiram o mínimo proposto para a saúde. Verificou-se uma maior proporção de adolescentes dos NSE D + E que não atendeu o critério recomendado. Estes achados demonstram que, aproximadamente, dois terços dos rapazes e um terço das moças apresentaram baixa aptidão cardiorrespiratória. Os adolescentes de NSE menos favorecidos foram os que apresentaram menores níveis de aptidão cardiorrespiratória.

\section{ABSTRACT}

\section{Cardiorespiratory fitness of adolescents from Florianópolis, SC}

Suitable levels of cardiorespiratory fitness during adolescence are closely concerned with a healthy lifestyle in adulthood. The objective of this study was to identify cardiorespiratory fitness behavior during adolescence and describe the prevalence of adolescents who fulfilled or not the healthy fitness criterion by sex, age and socioeconomic status (SES). The sample was composed by 963 adolescents (513 males and 450 females) aged 10-15 years old. A questionnaire was answered for attainment of the SES (A+ $B, C, D+E)$. To have the cardiorespiratory fitness measured, the PACER test (Progressive Aerobic Cardiovascular Endurance Run) was conducted. The number of laps completed in the test and the maximal oxygen uptake - $\dot{V} O_{2 \max }(\mathrm{ml} / \mathrm{kg} / \mathrm{min})$ were used as cardiorespiratory performance parameters. The statistical analysis

1. Núcleo de Pesquisa em Atividade Física e Saúde. Programa de PósGraduação em Educação Física, Universidade Federal de Santa Catarina.

Aceito em 13/6/07.

Endereço para correspondência: Daniel Giordani Vasques, Rua Vereador José do Vale Pereira, 48, apto. 204, Bairro Coqueiros - 88080-240 Florianópolis, SC, Brasil. E-mail: dgvasques@hotmail.com

\begin{abstract}
Palavras-chave: Aptidão física. Adolescente. Padrões de referência. Sexo. Fatores socioeconômicos.

Keywords: $\quad$ Physical endurance. Adolescent. Reference standards. Sex. Population characteristics.
\end{abstract}

included means and standard deviations, two-way variance analysis with Tukey post-hoc test, and chi-squared test ( $p<0.05)$. While the number of laps increased from 10 to 15 years, the $\dot{V O}_{2 \max }$ diminished, with no sex differences. Low SES adolescents presented lower fitness than the ones from the other SES. 68\% of males and $37.2 \%$ of females did not reach the healthy fitness criterion. It was verified the majority of the adolescents who did not fulfill the healthy fitness criterion was in the low SES. These findings show that, approximately $2 / 3$ of males and $1 / 3$ of females did not reach the healthy fitness criterion. Low SES adolescents presented the lowest levels of cardiorespiratory fitness.

\section{INTRODUÇÃO}

A aptidão cardiorrespiratória é um dos principais componentes da aptidão física relacionada à saúde ${ }^{(1)}$. Níveis adequados de aptidão cardiorrespiratória na adolescência associam-se inversamente a fatores de riscos cardiovasculares e metabólicos ${ }^{(2-4)}$, e estão diretamente relacionados a um aumento na participação em esportes, atividades físicas habituais, ingresso em profissões de elevadas exigências de atividade física e estilo de vida saudável na fase adulta da vida(5).

Levantamentos internacionais têm se preocupado em diagnosticar os níveis de aptidão cardiorrespiratória de adolescentes, e intervir quando estes valores mostram-se abaixo dos níveis considerados adequados para uma boa saúde ${ }^{(2-4)}$. Resultados de pesquisas têm mostrado que, durante a adolescência, a aptidão cardiorrespiratória dos rapazes é de 10 a 19\% maior que a das moças, variando em função da idade(5-11). Em relação aos critérios de classificação relacionados à saúde, observou-se que 37\% dos rapazes e $35 \%$ das moças norte-americanas não atendiam os critérios recomendados para a saúde(5), enquanto estudos com adolescentes do sul do Brasil identificaram cerca de 38-50\% dos rapazes e 55-57\% das moças com aptidão cardiorrespiratória abaixo do critério recomendado para a saúde ${ }^{(10,12)}$.

A relação do nível socioeconômico (NSE) com a aptidão cardiorrespiratória tem sido pouco documentada, principalmente no Brasil. Escolares que moravam em municípios com altos índices de desenvolvimento socioeconômico apresentaram aptidão cardiorrespiratória mais adequada à saúde do que aqueles de municípios de médio desenvolvimento socioeconômico, em ambos os se$x^{(13)}$. Estudo com adolescentes dinamarqueses não demonstrou diferenças entre o nível socioeconômico e o nível de aptidão física(14), porém, em moças norte-americanas, mostrou-se relação direta entre as classes socioeconômicas menos favorecidas e o baixo nível de aptidão física ${ }^{(15)}$.

Nesse contexto, é de fundamental relevância a realização de estudos que classifiquem a aptidão cardiorrespiratória de adolescentes brasileiros e que considerem além do sexo e da idade, o 
NSE. Desta forma, pode-se ter um melhor entendimento sobre as condições da aptidão cardiorrespiratória de adolescentes, a qual repercutirá na vida adulta. Esta pesquisa objetivou identificar o comportamento da aptidão cardiorrespiratória durante a adolescência e descrever a prevalência de adolescentes que atenderam e não atenderam o critério recomendado para a saúde por sexo, idade e NSE.

\section{MÉTODOS}

Caracterizado como uma pesquisa epidemiológica de corte transversal, o presente estudo é parte do projeto "Indicadores do Crescimento e Aptidão Física relacionada à Saúde de Escolares de Florianópolis-SC", desenvolvido de 2004 a 2006 em estudantes de escolas públicas e privadas. Os protocolos de intervenção do estudo foram aprovados pelo Comitê de Ética para Pesquisas com Seres Humanos da Universidade Federal de Santa Catarina (CEPSH/UFSC), sob o parecer 078/2003.

O processo amostral foi estratificado em três estágios: por região do município (centro, continente e interior), tipo de escola (pública e privada) e série de ensino (1a à 8a série). Dos estabelecimentos de ensino fundamental existentes no município, selecionou-se, sistematicamente, 11 escolas, sendo quatro estaduais, quatro municipais e três particulares. Na seqüência, sorteou-se, em cada escola, uma quantidade de turmas para se alcançar o número de sujeitos que representasse a região e o tipo de escola. Os dirigentes e professores das escolas sorteadas e os pais e filhos foram informadas sobre os objetivos do estudo e os procedimentos que seriam realizados. Após a autorização da direção e a obtenção do termo de consentimento livre e esclarecido, iniciouse a coleta de dados.

O tamanho amostral foi determinado a partir do modelo proposto por Luiz e Magnanini(16) para investigações epidemiológicas. Para efeito de cálculo considerou-se a prevalência de baixa aptidão física de 60\% e nível de confiança de 1,96 (Intervalo de Confiança $95 \%)$. A precisão adotada em torno da prevalência estimada foi de $3 \%$, sendo feito um acréscimo de $20 \%$ como possível índice de perda. Como a amostragem foi realizada por conglomerado, definiu-se um efeito do desenho igual a 1,5 e encontrou-se o número mínimo necessário de 1.214 escolares, porém, como foram avaliadas outras variáveis, a amostra total foi de 1.500 crianças e adolescentes.

Nesta pesquisa, optou-se por estudar adolescentes ${ }^{(17)}$, em função dos critérios de classificação do FITNESSGRAM(1) para a aptidão cardiorrespiratória relacionada à saúde, incluírem idades a partir dos 10 anos. Foram excluídos do estudo os escolares que não estavam presentes em sala de aula, que não entregaram o termo de consentimento livre e esclarecido e aqueles que se recusaram a participar da pesquisa $(n=71)$. Também não fizeram parte da amostra os que não se submeteram ao teste de aptidão cardiorrespiratória ( $n=24)$, que não informaram o sexo, a idade e o NSE ( $n=26)$ e aqueles com idade inferior a 10 anos ( $n=416)$.

$\mathrm{Na}$ coleta de dados, os escolares preencheram um questionário com informações demográficas e socioeconômicas, e foram submetidos a medições de massa corporal e estatura e à realização do teste $P A C E R^{(1)}$, além de outras medidas antropométricas e testes físicos não analisados neste estudo. Todas as medidas foram realizadas no ginásio de esporte da própria escola, em forma de circuito, com duração aproximada de 50 minutos por turma. A equipe de coleta de dados foi constituída por mestrandos e graduandos em Educação Física devidamente treinados.

O NSE foi medido com base no questionário da Associação Brasileira de Empresas de Pesquisa(18), que considera os bens de consumo familiar e o nível de escolaridade do chefe da família. A fim de facilitar a análise dos dados, os NSE foram agrupados em A + $B$ (mais favorecido), C (nível intermediário) e D + E (menos favorecido).
A aptidão cardiorrespiratória foi analisada a partir da realização do teste PACER (Progressive Aerobic Cardiovascular Endurance Run)(1), com reprodutibilidade de 0,87 a 0,93(20-22). O PACER é um teste de aptidão realizado com um aparelho sonoro, no qual a velocidade aumenta progressivamente a cada estágio (aproximadamente 1 minuto). Os escolares foram instruídos a correr uma distância de 20m, e a cada sinal sonoro emitido pelo aparelho de som, a ultrapassarem com um dos pés a linha demarcada, por quantas voltas fosse possível. O teste era encerrado quando o escolar parava por exaustão ou não conseguia manter a velocidade requerida por três voltas. O número de voltas completadas foi utilizado para análise.

O volume máximo de oxigênio expirado $\left(\dot{V}_{2 \max }\right)$ foi estimado (em $\mathrm{ml} / \mathrm{kg} / \mathrm{min}$ ) a partir da equação proposta por Léger et al.(20), que leva em consideração a velocidade (V) do avaliado no estágio em que o teste foi encerrado e a idade (ID). Estudos com adolescentes demonstraram correlações de validade do teste PACER para a estimativa do $\dot{\mathrm{V}} \mathrm{2}_{2 \max }$ de 0,51 a $0,83^{(20,22-24)}$, a partir da seguinte equação: $\dot{V} O_{2 \max }=31,025+(3,238 \times V)-(3,248 \times$ ID $)+$ $(0,1536 \times \vee \times I D)$.

Para classificar a aptidão cardiorrespiratória dos adolescentes, foram utilizados os pontos de corte recomendados para o teste PACER ${ }^{(1)}$, verificando os adolescentes que atenderam e os que não atenderam o critério recomendado para a saúde. Esta indicação se dá a partir do número de voltas realizadas pelo sujeito durante o teste e recomenda valores mínimos para estar na faixa recomendável para a saúde por sexo e idade (tabela 1).

\begin{tabular}{|c|c|c|c|c|c|c|}
\hline \multicolumn{7}{|c|}{$\begin{array}{c}\text { TABELA } 1 \\
\text { Número mínimo de voltas recomendado pelo } \\
\text { FITNESSGRAM }{ }^{(1)} \text { no teste } P A C E R \text { por sexo e idade }\end{array}$} \\
\hline & 10 anos & 11 anos & 12 anos & 13 anos & 14 anos & 15 anos \\
\hline Rapazes & 23 & 23 & 32 & 41 & 41 & 51 \\
\hline Moças & 15 & 15 & 23 & 23 & 23 & 23 \\
\hline
\end{tabular}

Na análise estatística, foram descritas e comparadas as médias dos indicadores da aptidão cardiorrespiratória (número de voltas e $\dot{\mathrm{V}} \mathrm{O}_{2 \max }$ ) dos adolescentes por sexo, idade e NSE, por meio da análise de variância two-way e do teste post-hoc de Tukey. Utilizou-se a freqüência percentual para classificar os adolescentes que atenderam e não atenderam o critério recomendado para a saúde por sexo, idade e NSE, e o teste qui-quadrado para comparar as proporções. Para todas as análises, adotou-se um nível de significância de $p<0,05$.

\section{RESULTADOS}

Participaram desse estudo 963 adolescentes, sendo 513 moças e 450 rapazes, com idades de 10 a 15 anos (11,9 $\pm 1,5$ anos), de escolas públicas e privadas do município de Florianópolis, SC. A figura $1 \mathrm{~A}$ apresenta os valores médios do $\dot{\mathrm{V}} \mathrm{O}_{2 \max }$ e do número de voltas dos adolescentes por idade. Foram observadas diferenças estatísticas entre as idades, sendo que, enquanto o desempenho no teste (número de voltas realizadas) aumentou dos 10 aos 15 anos $(p<0,001)$, o $\dot{V} O_{2 \max }$ teve decréscimo $(p<0,001)$. Não foram observadas diferenças significativas entre os sexos para o número de voltas e o $\dot{\mathrm{V}} \mathrm{O}_{2 \max }$ ao analisar a amostra total $(\mathrm{p}=0,072$ e $p=0,088$; respectivamente). Ao comparar os sexos e as idades conjuntamente, também não foram encontradas diferenças estatísticas ( $p=0,885$ e $p=0,879$; respectivamente) (figura 1B).

A freqüência percentual de adolescentes que atenderam e não atenderam o critério recomendado para a saúde ${ }^{(1)}$, por sexo e idade, é apresentada na figura 2. Os resultados demonstraram diferenças entre os sexos $(p<0,001)$, com maior proporção de moças 


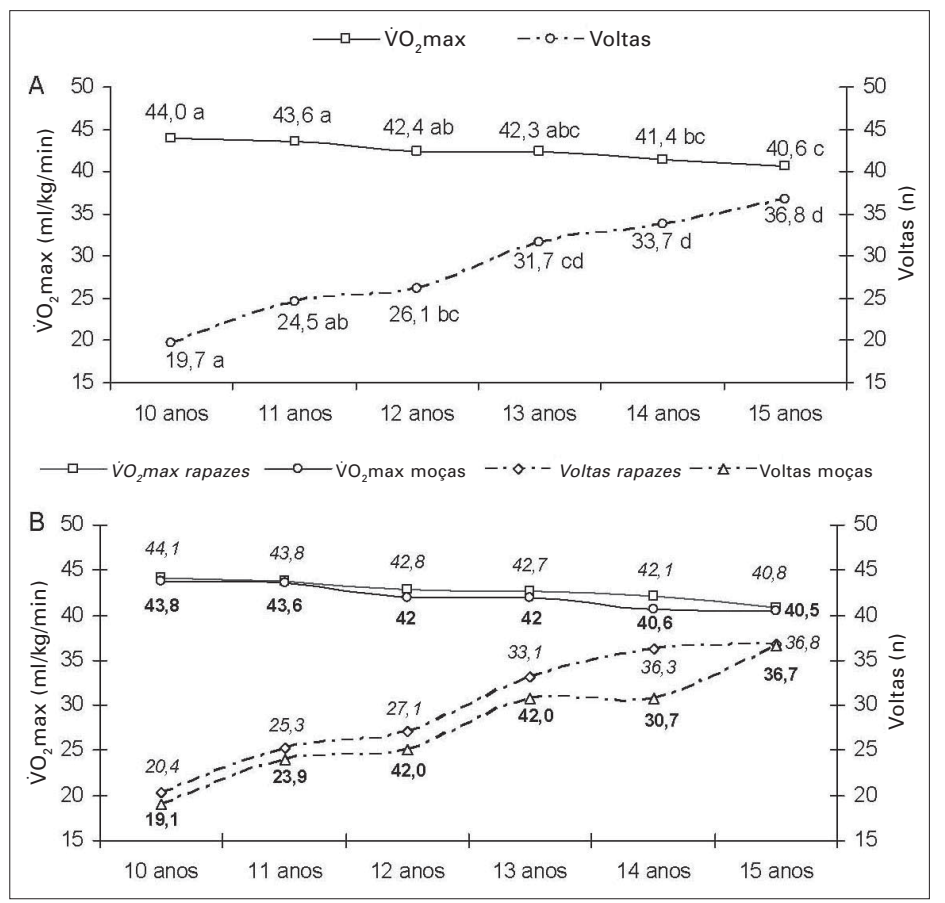

Figura 1 - Número de voltas e $\dot{V} O_{2 \max }$ dos adolescentes por idade lletras distintas indicam médias significativamente diferentes) (A) e por sexo e idade (B).
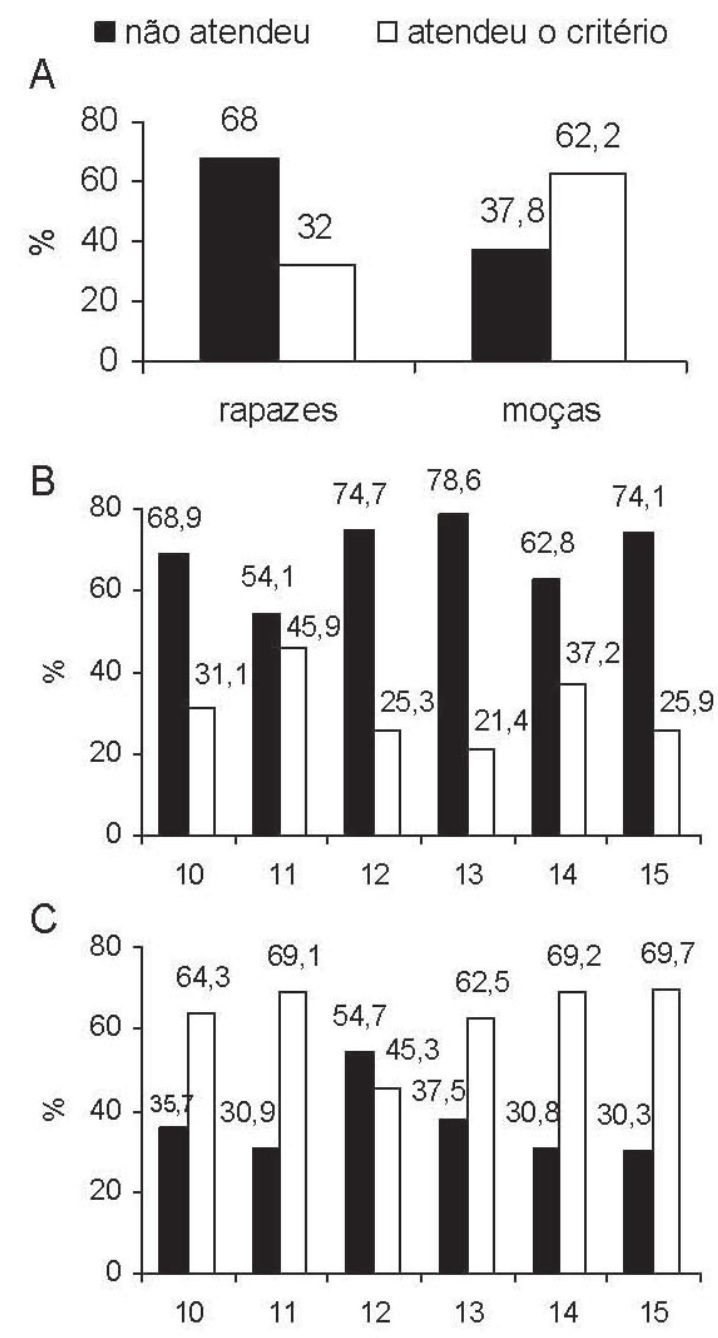

Figura 2 - Freqüencias percentuais de adolescentes que atenderam e não atenderam o critério(1), comparando-os entre os sexos $(\boldsymbol{A})$ e entre as idades para os rapazes (B) e para as moças (C).

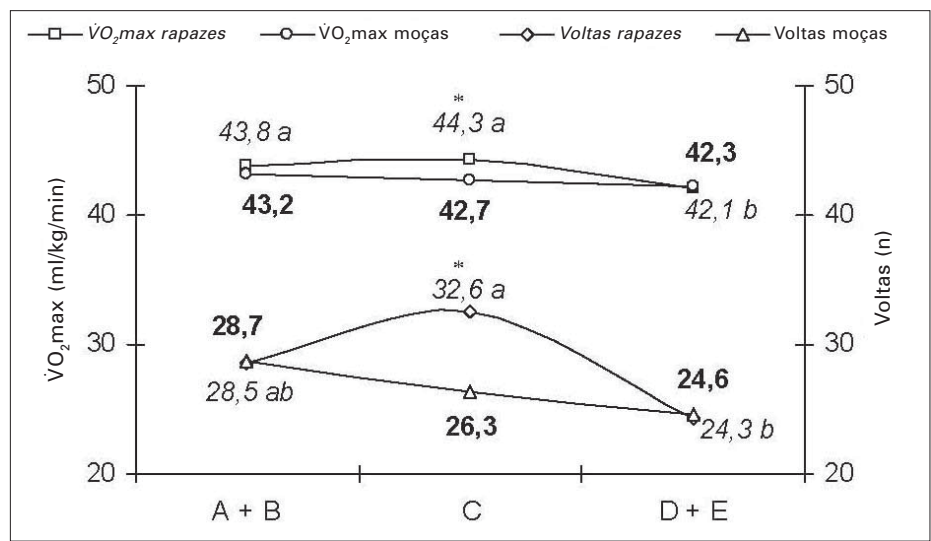

Figura 3 - Número de voltas e $\dot{V} O_{2 \max }$ dos adolescentes por sexo e NSE (* $p<0,05$ entre os sexos; letras distintas indicam médias significativamente diferentes entre os NSE).

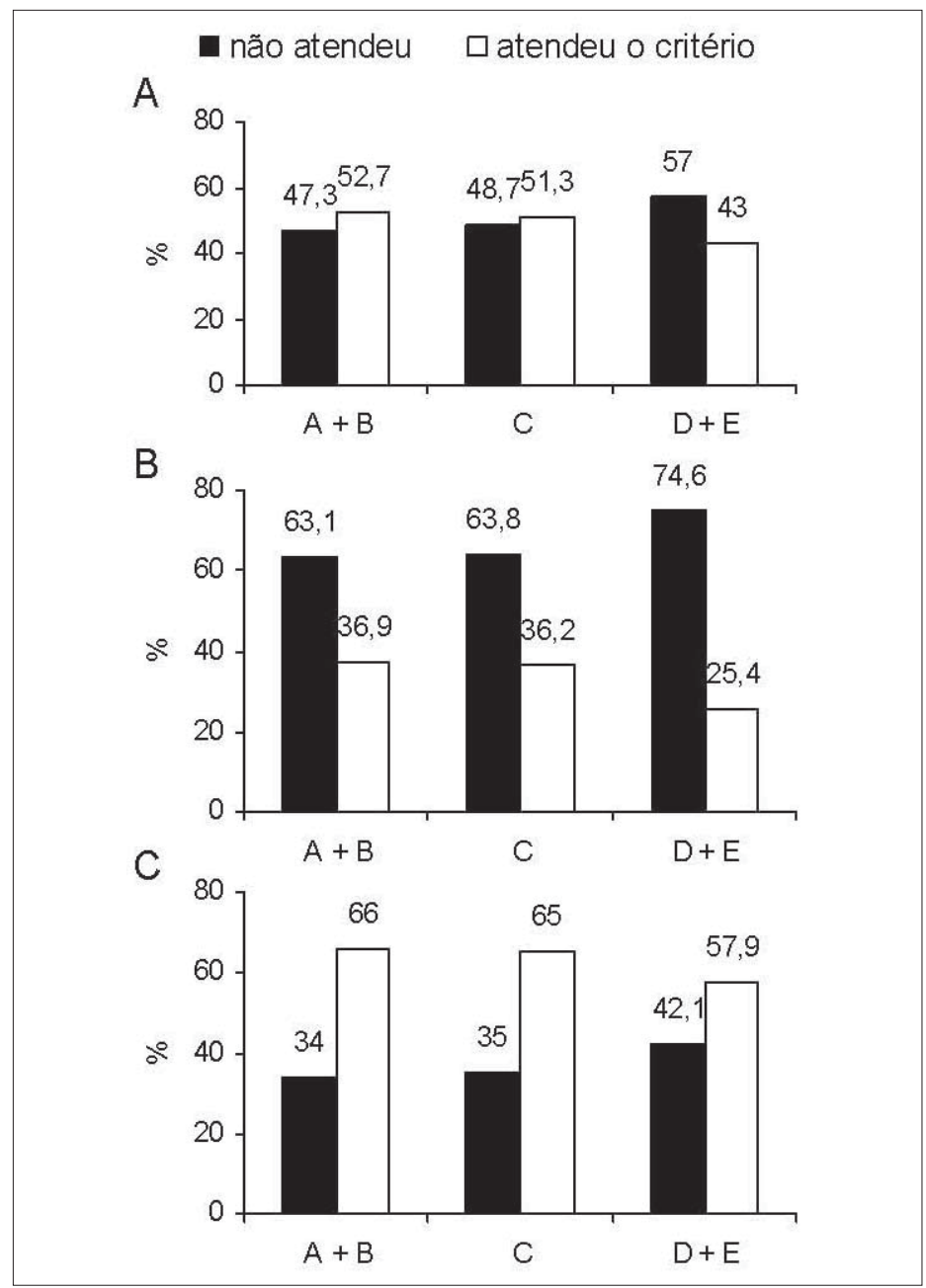

Figura 4 - Freqüências percentuais de adolescentes que atenderam e não atenderam o critério(1), a partir da comparação entre oS NSE (A), entre os rapazes $(\boldsymbol{B})$ e as moças $(\boldsymbol{C})$.

que atenderam, e de rapazes que não atenderam o critério recomendado para a saúde (figura 2A). Nos rapazes, ocorreu maior proporção de sujeitos que não atenderam o critério nas idades de 12,13 e 15 anos e menor proporção na idade de 11 anos ( $p=$ $0,004)$. Pode-se observar em todas as idades, que mais de $50 \%$ dos rapazes não atendeu o critério (figura 2B). Verificou-se maior proporção de moças que não atenderam a recomendação aos 12 anos e que atenderam nas demais idades ( $p=0,007)$, com valores mais acentuados aos 11, 14 e 15 anos (figura 2C). 
Verificaram-se diferenças entre as médias dos NSE, tendo os escolares dos NSE A + B e C obtido valores do número de voltas e do $\dot{V}_{2 \max }$ superiores àqueles dos NSE D + E $(p<0,001)$. Observaram-se diferenças estatísticas ao analisar o sexo e o NSE ( $p=$ 0,021 para o número de voltas e $\mathrm{p}=0,044$ para o $\dot{\mathrm{VO}}_{2 \text { max }}$ ), sendo que os rapazes do NSE $C$ obtiveram valores superiores às moças no $\dot{\mathrm{V}} \mathrm{O}_{2 \max }$ e no número de voltas. Notou-se ainda que os rapazes dos NSE A + B e C obtiveram valores do $\dot{\mathrm{VO}}_{2 \max }$ significativamente superiores aos dos NSE D + E, enquanto que no número de voltas, os rapazes do NSE C foram superiores aos dos NSE D + E (figura 3). Não foram encontradas relações significativas entre o NSE e a idade $(p>0,05)$.

Ao analisar a aptidão cardiorrespiratória dos adolescentes a partir dos NSE, observou-se maior proporção de adolescentes (57\%, $p=0,03$ ) que não atenderam o critério recomendado para a saúde nos NSE menos favorecidos ( $+E$ ) (figura 4A). Por outro lado, mais da metade dos adolescentes classificados nos NSE A + B e $C$ atenderam o critério recomendado para a saúde. Em relação aos sexos (figuras 4B e 4C), não foram encontradas diferenças estatísticas entre o NSE e a aptidão cardiorrespiratória nos rapazes ( $p=0,053)$ e nas moças $(p=0,244)$.

\section{DISCUSSÃO}

Os resultados mostraram aumento no desempenho no teste (número de voltas) à medida que a idade evoluía. Esse comportamento também foi observado em adolescentes norte-americanos, principalmente dos 11 aos 15 anos para os rapazes e dos 11 aos 12 para as moças ${ }^{(25)}$. Em escolares de Marechal Cândido Rondon$P R$, foi observado aumento progressivo no desempenho do teste PACER dos 7 aos 17 anos para ambos os sexos ${ }^{(10)}$. Alguns fatores que explicam essa concordância entre os estudos são as mudanças que ocorrem no sistema cardiorrespiratório e na composição corporal, em função do crescimento e desenvolvimento físico, facilitando, assim, um melhor desempenho(6-7,11,26).

Apesar de o número de voltas realizadas ter aumentado dos 10 aos 15 anos, o $\dot{V} O_{2 \max }$ diminuiu. Wilmore e Costill(7) citam que o $\dot{\mathrm{VO}}_{2 \max }$ relativo $(\mathrm{ml} / \mathrm{kg} / \mathrm{min})$ tende a se manter para os rapazes dos 6 anos à vida adulta e a diminuir para as moças a partir dos 13 anos de idade. Em jovens norte-americanos, a aptidão cardiorrespiratória dos rapazes aumentou dos 12 aos 19 anos, enquanto a das moças declinou(5). Em outro estudo norte-americano foi observada queda do $\dot{\mathrm{VO}}_{2 \max }$ relativo dos 8 aos 16 anos em ambos os $\operatorname{sexos}^{(8)}$. No Brasil, estudo com escolares sergipanos de 7 a 14 anos identificou manutenção do $\dot{\mathrm{V}} \mathrm{O}_{2 \max }$ para os rapazes e queda para as meninas até os 11 anos de idade ${ }^{(9)}$, enquanto em escolares de Marechal Cândido Rondon-PR foi observada queda nos valores do $\dot{\mathrm{VO}}_{2 \max }$ em ambos os sexos e em todas as idades (7-17 anos) $)^{(10)}$.

Nos estudos internacionais analisados ${ }^{(5,7-8)}$, ocorreu uma convergência de declínio maior nas moças do que nos rapazes em função do aumento da idade. No Brasil, também se observou essa queda nas meninas sergipanas ${ }^{(9)}$, em ambos os sexos em uma cidade do Paraná(10), e neste estudo quando analisados os sexos conjuntamente. Esse declínio evidenciado com o aumento da idade acontece em função do aumento da massa corporal que ocorre na adolescência, já que quanto maior a massa corporal, menor o $\dot{\mathrm{V}}_{2 \max }$ relativo, medido em mililitros de oxigênio por quilograma de peso por minuto $(\mathrm{ml} / \mathrm{kg} / \mathrm{min})$. Possivelmente, o aumento da massa corporal foi maior do que o aumento no volume de oxigênio (em I/min), fazendo com que o $\dot{V}_{2 \max }$ relativo diminuísse com a idade. Outros fatores como a redução do nível de prática de atividade física na fase da adolescência, principalmente nas meninas ${ }^{(27)}$, e os períodos diferenciados de maturação podem ter influenciado os resultados.

No presente estudo, os valores médios do número de voltas e do $\dot{V} \mathrm{O}_{2 \max }$ não diferiram entre os sexos. Diversos estudos têm mostrado que, durante a adolescência, o desempenho no teste e o $\dot{\mathrm{V}}{ }_{2 \max }$ das moças, medido direta ou indiretamente, são menores que os dos rapazes ${ }^{(5,6,8-11,28)}$. Provavelmente, este fato não ocorreu neste estudo em decorrência dos baixos valores atingidos pelos rapazes que obtiveram valores médios do $\dot{\mathrm{V}} \mathrm{O}_{2 \max }$ apenas 1,92\% maiores que o das moças, sendo esta diferença um pouco maior aos 14 e 15 anos (3,59\%). Estudos também têm apresentado aumento destas diferenças entre os sexos dos 10 aos 15 anos, porém em valores consideravelmente maiores (10 a 19\%)(5,7).

Foi observada menor quantidade de rapazes com níveis adequados de aptidão cardiorrespiratória para a saúde em relação às moças (32\% e 62,2\%, respectivamente). No estudo com jovens norte-americanos, $62,6 \%$ dos rapazes e $65,3 \%$ das moças superaram o mínimo proposto(5). Em escolares paranaenses observouse que quase $50 \%$ do rapazes e $43 \%$ da moças de 10 a 17 anos atenderam o critério proposto(10), enquanto em escolares gaúchos de 7 a 17 anos, encontrou-se $52 \%$ dos rapazes e $43 \%$ das moças acima do mínimo(28). Apesar das diferenças encontradas em relação aos sexos, pode-se constatar uma situação preocupante com os níveis de aptidão apresentados pelos rapazes de FlorianópolisSC. Acredita-se que baixos níveis de atividade física, maiores valores de gordura corporal e maturação tardia possam ter dificultado para os rapazes melhores desempenhos no teste.

Quando comparadas as idades, verificou-se maior proporção de rapazes que atenderam o critério recomendado para a saúde aos 11 anos e menor aos 12, 13 e 15 anos de idade. Nas moças, foi observada maior proporção atendendo o critério aos 11, 14 e 15 anos e menor aos 12 anos. Jovens paranaenses de 15 a 17 anos apresentaram maior proporção de sujeitos que não atendeu o critério recomendado do que os de 7-14 anos (41,5\% rapazes e 30,2\% moças) ${ }^{(10)}$. Adolescentes norte-americanos demonstraram menor proporção atendendo o critério recomendado aos 12 e 13 anos $(55,3 \%)$ do que dos 14 aos 19 anos $(70 \%)^{(5)}$. Os resultados não mostraram uma tendência de aumento ou declínio da aptidão com a idade, talvez em função das diferenças metodológicas como testes e critérios de classificação adotados, assim como o reduzido número de estudos na literatura considerando a idade.

Em relação ao NSE, verificou-se menor aptidão nos adolescentes dos NSE menos favorecidos ( $D+E$ ). Constatou-se também nos NSE mais baixos, maior proporção de adolescentes que não atenderam o critério recomendado para a saúde. Em escolares gaúchos de 7 a 17 anos com índice de desenvolvimento socioeconômico médio, foi verificada maior proporção de sujeitos que não atenderam o critério recomendado quando comparados aos pertencentes a municípios com alto índice ${ }^{(13)}$. O mesmo foi observado em moças norte-americanas, sendo apontada como possível causa a maior prevalência de sobrepeso e obesidade ${ }^{(15)}$. Em estudo com escolares dinamarqueses, não foram verificadas diferenças na aptidão cardiorrespiratória e no IMC entre os diferentes NSE ${ }^{(14)}$. No presente estudo, observou-se que a média do IMC dos adolescentes dos NSE D + E foi menor que os demais NSE (dados não apresentados). Possivelmente, baixos níveis nutricionais e de atividade física tenham influenciado o menor desempenho destes adolescentes ${ }^{(29)}$.

Dentre as limitações desse estudo, um fator importante a ser citado é que o desempenho no teste pode ser alterado pelo nível de motivação e espírito de competitividade. Além disso, a variação no nível de atividade física, estágio maturacional e composição corporal também podem ter afetado os resultados. Sugere-se que estudos futuros analisem a aptidão cardiorrespiratória controlando essas variáveis. $\mathrm{O} \mathrm{V}_{2 \max }$ foi estimado a partir de um teste indireto, no entanto, este tipo de teste é mais recorrido em estudos populacionais por apresentar baixo custo de realização e praticidade na avaliação.

Conclui-se que enquanto o desempenho no teste PACER aumenta dos 10 aos 15 anos, o $\dot{V} O_{2 \max }$ relativo ao peso corporal diminui, principalmente devido ao aumento da massa corporal durante 
a adolescência. Em relação ao desempenho no teste, dois terços dos rapazes e um terço das moças não atenderam o mínimo recomendado para a saúde. A aptidão cardiorrespiratória dos adolescentes pertencentes aos NSE mais baixos foi menor quando comparada aos de NSE intermediário e elevado. As estratégias de intervenção para este grupo devem atingir, principalmente, os adolescentes do sexo masculino e de condição socioeconômica menos favorecida.

Todos os autores declararam não haver qualquer potencial conflito de interesses referente a este artigo.

\section{REFERÊNCIAS}

1. Cooper Institute for Aerobics Research (CIAR). The prudential FITNESSGRAM test administration manual. Dallas: Author; 1999.

2. Twisk JWR, Kemper HCG, Van Mechelen W. The relationship between physical fitness and physical activity during adolescence and cardiovascular disease risk factors at adult age. The Amsterdam growth and health longitudinal study. Int $J$ Sports Med. 2002;23:8-14

3. Janz KF, Dawson JD, Mahoney LT. Increases in physical fitness during childhood improve cardiovascular health during adolescence: the Muscatine study. Int J Sports Med. 2002;23:15-21.

4. Lefevre J, Philippaerts R, Delvaux K, Thomis M, Claessens AL, Lysens R, et al. Relation between cardiovascular risk factors at adult age, and physical activity during youth and adulthood: the leuven longitudinal study on lifestyle, fitness and health. Int J Sports Med. 2002;23:32-8.

5. Pate RR, Wang CY, Dowda M, Farrell SW, O'Neill JR. Cardiorespiratory fitness levels among US youth 12 to 19 years of age: findings from the $1999-2002 \mathrm{Na}$ tional Health and Nutrition Examination Survey. Arch Pediatr Adolesc Med. 2006; 160(10):1005-12

6. Rowland T, Goff D, Martel L, Ferrone L. Influence of cardiac functional capacity on gender differences in maximal oxygen uptake in children. Chest. 2000:117: 629-35.

7. Wilmore JH, Costill DL. Fisiologia do esporte e do exercício. Manole; 2001

8. McMurray RG, Harrell JS, Bradley CB, Deng S, Bangdiwala SI. Predicted maximal aerobic power in youth is related to age, gender, and ethnicity. Med Sci Sports Exerc. 2002;34(1):145-51.

9. Silva RJS. Características de crescimento, composição corporal e desempenho físico relacionado à saúde de crianças e adolescentes de 7 a 14 anos da região de Cotinguiba/SE. Dissertação de Mestrado - Mestrado em Educação Física: Universidade Federal de Santa Catarina; 2002.

10. Hobold E. Indicadores de aptidão física relacionada à saúde de crianças e adolescentes do município de Marechal Cândido Rondon - Paraná, Brasil. Dissertação de Mestrado - Mestrado em Educação Física: Universidade Federal de Santa Catarina; 2003.
11. Geithner CA, Thomis MA, Eynde BV, Maes HHM, Loos RJF, Peters M, et al. Growth in peak aerobic during adolescence. Med Sci Sports Exerc. 2004;39(9): 1616-24.

12. Glaner MF. Aptidão física relacionada à saúde de adolescentes rurais e urbanos em relação a critérios de referência. Rev Bras Educ Fís Esp. 2005;19(1):13-24.

13. Marafiga D, Gaya A, Torres L, Garlipp D, Moreira R, Silva M. Associação entre aptidão relacionada à saúde e o índice de desenvolvimento sócio econômico em escolares de municípios do Rio Grande do Sul. Revista Perfil. 2005;7(7):60-7

14. Kristensen PL, Wedderkopp N, Moller NC, Andersen LB, Bai CN, Froberg K. Tracking and prevalence of cardiovascular disease risk factors across socio-economic classes: a longitudinal substudy of the European Youth Heart Study. BMC Public Health. 2006;27:6-20

15. Fahlman MM, Hall HL, Lock R. Ethnic and socioeconomic comparisons of fitness, activity levels, and barriers to exercise in high school females. J Sch Health. 2006;76(1):12-7.

16. Luiz RR, Magnanini MMF. A lógica da determinação do tamanho da amostra em investigações epidemiológicas. Cad Saúde Coletiva 2000;8(2):9-28.

17. WHO (World Health Organization). Physical status: the use and interpretation of anthropometry: report of a WHO Expert Committee. Geneva; 1995.

18. ABEP - Associação Brasileira de Empresas de Pesquisa. Dados com base no Levantamento Sócio Econômico - 2000. Disponível em <www.abep.org>. Acessada em 20/11/2006, 2003

19. Gordon CC, Chumlea WC, Roche AF. Stature, recumbent length and weight. In: Lohman TG, Roche AF, Martorell R, editors. Anthropometric standardization reference manual. Champaign, IL: Human Kinetics; 1991.

20. Léger LA, Mercier D, Gadoury C, Lambert J. The multistage 20-meter shuttle run test for aerobic fitness. J Sports Sci. 1988;6:93-101.

21. Duarte CR, Duarte MFS, Andrade DR. Teste aeróbico de Luc Léger - aplicabilidade para a realidade brasileira. Anais do XVII Simpósio Internacional de Ciências do Esporte, São Caetano do Sul, SP; 1990. p. 87

22. Liu NYS, Plowman SA, Looney MA. The reliability and validity of the 20-meter shuttle run test in American students 12 to 15 years old. Res Q Exerc Sport. 1992;63(4):360-5.

23. Mahoney C. 20-MST and PWC170 validity in non-Caucasian children in the UK (abstract). Br J Sports Med. 1992;26:45-7.

24. Suminski RR, Ryan ND, Poston CS, Jackson AS. Measuring aerobic fitness of Hispanic youth 10 to 12 years of age. Int J Sports Med. 2004;25:61-7.

25. Beets MW, Pitetti KH, Cardinal BJ. Progressive aerobic cardiovascular endurance run and body mass index among an ethnically diverse sample of 10-15-year-olds. Res Q Exerc Sport. 2005;76:389-97.

26. Malina RM, Bouchard C, Bar-Or O. Growth, maturation, and physical activity. Champaign, IL: Human Kinetics; 2004.

27. Twisk JWR. Physical activity guidelines for children and adolescents: a critical review. Sports Med. 2001;31(8):617-27.

28. Bergmann G, Lorenzi T, Garlipp D, Marques AC, Araújo M, Lemos A, et al. Aptidão física relacionada à saúde de crianças e adolescentes do Estado do Rio Grande do Sul. Revista Perfil. 2005;7(7):12-21.

29. Monyeki MA, Koppes LLJ, Kemper HCG, Monyeki KD, Toriola AL, Pienaar AE, et al. Body composition and physical fitness of undernourished South African rural primary school children. Eur J Clin Nutr. 2005;59:877-83. 